\title{
A SPECTRAL CHARACTERIZATION OF THE $s$-CLIQUE EXTENSION OF THE TRIANGULAR GRAPHS
}

\author{
YING-YING TAN \\ School of Mathematics \& Physics \\ Anhui Jianzhu University, Hefei, Anhui, 230201, PR China \\ e-mail: tansusan1@ahjzu.edu.cn \\ JACK H. KOOLEN ${ }^{1}$ \\ School of Mathematical Sciences \\ University of Science and Technology of China, Hefei, Anhui, 230026, PR China \\ Wen-Tsun Wu Key Laboratory of the CAS, School of Mathematical Sciences \\ University of Science and Technology of China, Hefei, Anhui, 230026, PR China \\ e-mail: koolen@ustc.edu.cn \\ AND \\ ZHENG-JiANG XIA \\ School of Finance, Anhui University of Finance and Economics \\ Bengbu, Anhui, 233030, PR China \\ e-mail: xzj@mail.ustc.edu.cn \\ This paper is dedicated to the memory of Prof. Slobodan Simić.

\begin{abstract}
A regular graph is co-edge regular if there exists a constant $\mu$ such that any two distinct and non-adjacent vertices have exactly $\mu$ common neighbors. In this paper, we show that for integers $s \geq 2$ and $n$ large enough, any coedge-regular graph which is cospectral with the $s$-clique extension of the triangular graph $T(n)$ is exactly the $s$-clique extension of the triangular graph $T(n)$.
\end{abstract}

Keywords: co-edge-regular graph, $s$-clique extension, triangular graph.

2010 Mathematics Subject Classification: 05C50, 05C75, 05C62.

\footnotetext{
${ }^{1}$ Corresponding author.
} 


\section{REFERENCES}

[1] A.E. Brouwer, A.M. Cohen and A. Neumaier, Distance-Regular Graphs (SpringerVerlag, Berlin, 1989).

doi:10.1007/978-3-642-74341-2

[2] A.E. Brouwer and W.H. Haemers, Spectra of Graphs (Springer, Heidelberg, 2012). doi:10.1007/978-1-4614-1939-6

[3] L.C. Chang, The uniqueness and non-uniqueness of the triangular association scheme, Sci. Record 3 (1959) 604-613.

[4] S. Hayat, J.H. Koolen and M. Riaz, A spectral characterization of the s-clique extension of the square grid graphs, European J. Combin. 76 (2019) 104-116. doi:10.1016/j.ejc.2018.09.009

[5] C.D. Godsil, G. Royle, Algebraic Graph Theory (Springer-Verlag, Berlin, 2001). doi:10.1007/978-1-4613-0163-9

[6] W.H. Haemers, Interlacing eigenvalues and graphs, Linear Algebra Appl. 226-228 (1995) 593-616. doi:10.1016/0024-3795(95)00199-2

[7] A.J. Hoffman, On the polynomial of a graph, Amer. Math. Monthly 70 (1963) $30-36$. doi:10.1080/00029890.1963.11990038

[8] J.H. Koolen, B. Gebremichel and J.Y. Yang, Sesqui-regular graphs with fixed smallest eigenvalue. https://arxiv.org/abs/1904.01274v1

[9] P. Terwilliger, Algebraic Graph Theory, Lecture Notes, unpublished. https://icu-hsuzuki.github.io/lecturenote/

[10] E.R. van Dam, Regular graphs with four eigenvalues, Linear Algebra Appl. 226-228 (1995) 139-162. doi:10.1016/0024-3795(94)00346-F

[11] E.R. van Dam, J.H. Koolen and H. Tanaka, Distance-regular graphs, Electron. J. Combin. (2016) \#DS22.

[12] J.Y. Yang and J.H. Koolen, On the order of regular graphs with fixed second largest eigenvalue. http://arxiv.org/abs/1809.01888v1

Received 7 May 2019

Revised 8 August 2019

Accepted 9 August 2019 